\title{
Mucosal Fixed Drug Eruption to Levetiracetam with Early Positive Patch Test on Non-Lesional Skin
}

Nazli Ercan ${ }^{1}$

${ }^{1}$ University of Health Sciences Turkey

November 3, 2021

\begin{abstract}
Levetiracetam (LEV) is a second-generation antiepileptic drug (AED) that is well tolerated, has a broad spectrum of action, low protein binding, and minimal hepatic metabolism. The incidence of hypersensitivity to LEV in children and adults is $0.6 \%$. This is the first reported fixed drug eruption (FDE) identified using a patch test in a pediatric case associated with LEV
\end{abstract}

\section{Hosted file}

LEV-FDE Main Document 31.10.2021.docx available at https://authorea.com/users/444401/ articles/544144-mucosal-fixed-drug-eruption-to-levetiracetam-with-early-positive-patchtest-on-non-lesional-skin

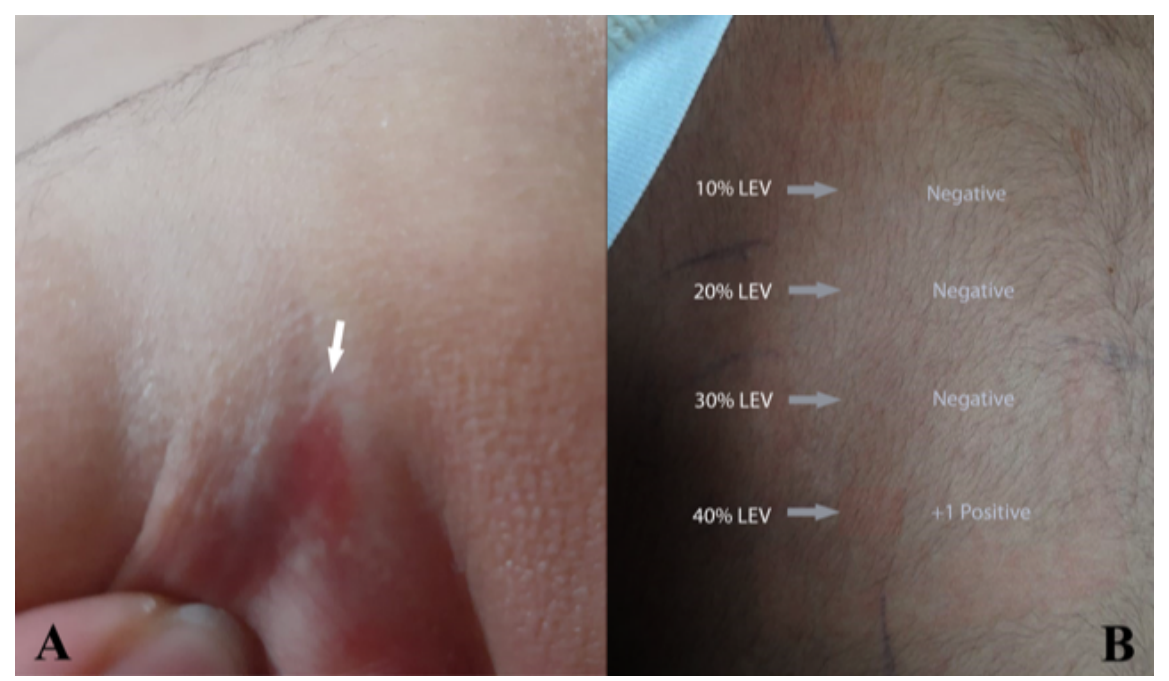

